\title{
Peak Height Precision in Hadamard Transform Time-of-Flight Mass Spectra
}

\author{
Joel R. Kimmel, ${ }^{* \ddagger}$ Oh Kyu Yoon, ${ }^{*}$ Ignacio A. Zuleta, Oliver Trapp, ${ }^{\dagger}$ \\ and Richard N. Zare \\ Department of Chemistry, Stanford University, Stanford, California, USA
}

\begin{abstract}
Hadamard transform (HT) time-of-flight mass spectrometry (TOFMS) is a multiplexing technique that offers high duty cycle for the mass analysis of continuous ion sources. The multiplexing advantage is maximized when spectral noise is independent of signal intensity. For conditions in which shot noise predominates, the variance in each peak is a function of the population of all measured species. We develop expressions for the performance of a HT-TOF mass spectrometer based on Poissonian statistics for the arrival times of ions at the detector. These expressions and complementary probabilistic simulations are used to estimate the magnitude of the baseline noise as a function of mass spectral features and acquisition conditions. Experiment validates the predictions that noise depends on the total number of ions in the acquired spectrum, and the achieved signal-to-noise ratio for a given species depends on its relative population. We find that for HT-TOFMS experiments encoded with an $n$-order binary off-on sequence that contains $N=2^{n}-1$ elements, the peak height precision, which is the peak intensity divided by its standard deviation, is greater than that of an equivalent conventional TOF experiment by a factor of $\sqrt{N / 2}$ times the square root of the fractional abundance of the peak of interest. Thus, HT-TOFMS is superior to conventional TOF for all species whose fractional abundance $F_{i}$ exceeds $2 / N$, which for a typical $N$ value of 2047 corresponds to $F_{i}>0.001$. HT-TOF mass spectra collected at 2500 per second demonstrates the method's capability of monitoring transient processes not possible by conventional means. (J Am Soc Mass Spectrom 2005, 16, 1117-1130) (c) 2005 American Society for Mass Spectrometry
\end{abstract}

$\mathrm{H}$ adamard transform time-of-flight mass spectrometry (HT-TOFMS) [1, 2] is based on the pseudorandom gating of ion packets into a time-of-flight analyzer. In its typical implementation, the technique is able to monitor continuous ion sources with a $50 \%$ duty cycle, by which we mean the sample utilization efficiency, independent of all other figures of merit. Recently, we have demonstrated that the duty cycle can be extended to $100 \%$ using patterned, twochannel detection [3]. While a conventional TOFMS experiment releases a single packet of ions and makes independent measurements of all species, an HTTOFMS experiment releases thousands of packets and measures the intensities of species as linear sums. For a fixed acquisition time, this procedure leads to linear increases in signal intensities and a root mean square reduction in the relative magnitude of signal-indepen-

Published online June 4, 2005

Address reprint requests to Professor R. N. Zare, Department of Chemistry, Stanford University, Room 133, S. G. Mudd Bldg., Stanford, CA 94305-5080, USA. E-mail: zare@stanford.edu

* These authors contributed equally to this work.

'Present address: Max-Planck-Institut für Kohlenforschung, KaiserWilhelm-Platz 1, 45470 Mülheim an der Ruhr, Germany.

‡ Present address: Cooperative Institute for Research in the Environmental Sciences, University of Colorado, Boulder, Colorado, 80309-0216. dent noise. Signal-dependent noise is known to affect the achieved performance of HT-TOFMS [4], but these processes have not been carefully studied.

The principles of HT-TOFMS have been presented in previous publications [1-3]. Briefly, a continuous, accelerated ion beam is modulated on and off the axis of detection following a binary, pseudorandom encoding sequence that is applied at $\mathrm{MHz}$ rates. Because the produced ion packets have nanosecond widths, and typical flight times are on the order of tens or hundreds of microseconds, thousands of ion packets drift through the flight chamber at any given moment (compared to one packet in conventional TOFMS). Ions from these packets interpenetrate one another as they fly. Consequently, the detected signal corresponds to the sum of the time-shifted spectra of the individual packets. With knowledge of the applied encoding sequence, the acquired data set is mathematically deconvoluted to recover the time-of-flight mass spectrum.

A Bradbury-Nielson gate (BNG) [5] modulates the ion beam at the entrance of the field-free drift region of the mass spectrometer. The BNG consists of two finely spaced, interleaved sets of wire electrodes that are electrically isolated from one another and lie in a plane perpendicular to the trajectory of the ion beam. Encoding sequences are applied to the ion beam by alternat- 
ing the voltage of the electrodes between two states: a sequence element 1 fixes the gate electrodes at relative ground and allows ions to pass undeflected, whereas a sequence element 0 shifts the gate electrodes to a deflecting state in which the voltage of one wire set is raised to a positive bias as the voltage of the other is lowered to a negative bias of equal magnitude. The deflection causes the ions to miss the detector at the plane of the detector.

Ion beam encoding in HT-TOFMS follows simplextype sequences [6], with length based on the range of flight times being monitored and the temporal width of the applied on-off encoding elements. Typical sequences for HT-TOFMS have 1023, 2047, or 4095 elements with widths between 50 and 200 ns. Because all sequences of this type contain an approximately equal number of ones and zeros (i.e., transmission and deflection elements), the duty cycle of any one-channel HTTOFMS experiment is $50 \%$.

For conditions where the noise is independent of signal intensity, simplex encoding reduces the relative time-independent noise by a factor that depends on the number of encoding elements in the applied sequence. If a TOF spectrum consisting of $N$ acquisition elements is measured by using an $N$-element simplex sequence, the predicted signal-to-noise ratio improvement over the equivalent conventional TOF experiment, termed the multiplexing or Felgett advantage, is given by [6]:

$$
\frac{\mathrm{SNR}^{\mathrm{HT}}}{\mathrm{SNR}^{\mathrm{TOF}}}=\frac{\sqrt{N}}{2}
$$

where SNR is the ratio of signal intensity to the standard deviation of the baseline. For such conditions, the multiplexed TOFMS scheme should always increase sensitivity and speed for the analysis of continuous beams of ions.

Simplex-type multiplexing strategies have also been used to increase the sensitivity of spectroscopic experiments [7-10]. It has been shown that the achieved SNR improvement in these experiments depends strongly on the nature of spectral noise. For systems where error is dominated by intensity-dependent noise, such as shot noise or source fluctuations, the multiplexing advantage decreases as the number of bright features increases.

The noise observed in HT-TOF mass spectra has both random and discrete components. Discrete noise, which results from time-invariant inaccuracies in the applied encoding sequence, appears as positive and negative spikes with intensities that are proportional to the magnitude of the encoding error and the intensity of the true peak from which it originates [4]. These effects are minimized by ensuring that applied encoding sequences have a square shape and modulate the beam between two discrete states. In what follows, we assume that discrete noise is negligible.

Random baseline noise in deconvoluted HT-TOF mass spectra also appears with positive and negative intensity. In previous work [11], we demonstrated that the root mean square of this baseline varies linearly with the square root of the intensity of all ions in the spectrum. It was suggested that this baseline likely originated from fluctuations in the recorded signal intensities. This signal-dependent behavior implies that $\mathrm{SNR}^{\mathrm{HT}}$ will deviate from that predicted by eq 1 in a fashion similar to that observed in HT spectroscopy. Thus, in the worst case scenario, the multiplexing scheme may actually produce a poorer SNR than conventional TOFMS. We explore when this condition might occur and when advantages in the SNR of the HT-TOFMS do exist.

HT-TOFMS is an ion counting technique, which uses a voltage-discriminated multichannel scaler (MCS) to digitize the output of the multichannel plate (MCP) detector. Based on observations of the recorded signal with no ion current present, uncorrelated detector noise (e.g., thermal noise or dark counts) is considered to be negligible. The pulses from the MCP in response to incident ions have a distribution of heights. Within each acquisition time bin, the MCS registers any pulse exceeding the discriminator threshold as a single ion count. Thus, mass spectra are insensitive to the pulse height distribution. Likewise, any signals with intensity less than the discriminator threshold are ignored so that MCP ringing presents no contribution. Therefore, the primary cause of the observed baseline variation in HT-TOF mass spectra is fluctuations in the current of the ion beam on the timescale of the acquisition process.

This threshold detection strategy relies on low ion currents so that the frequency of simultaneous ion arrivals is inconsequential. Unlike analog detection systems, where the height or integral of detector response yields information about the abundance of species, the relative peak intensities of the various species within a spectrum collected by ion counting arise from the summing of multiple measurements of each species. In conventional TOFMS, this task is accomplished by averaging multiple passes. In HT-TOFMS, a single pass records $N / 2$ measurements of each ion species, and therefore fewer passes should be required to obtain precise peak heights.

Modulation of the ion beam produces the $N$-element column vector $\mathbf{y}$, which represents the recorded data. Mathematically, this physical convolution is equivalent to

$$
\mathbf{y}=\mathbf{S x}+\varepsilon
$$

where $\mathbf{S}$ is the $N \times N$ simplex matrix, $\mathbf{x}$ is the $N$-element column vector with values representing the true population of ion species that would arrive at the detector during each element of $\mathbf{y}$ without encoding of the ion beam, and $\boldsymbol{\varepsilon}$ is an $N$-element column vector composed of signal-independent noise. Recall that under standard conditions, $\boldsymbol{\varepsilon}=0$. To recover the time-of-flight spectrum $\mathbf{z}$, the acquired data set $\mathbf{y}$ is deconvoluted with the inverse of $\mathbf{S}$, i.e., 


$$
\mathbf{z}=\mathbf{S}^{-1} \mathbf{y}
$$

At first, it might be thought from eqs 2 and 3 that the HT-TOFMS technique recovers exactly the time-offlight mass spectrum when $\boldsymbol{\varepsilon}=\mathbf{0}$. In reality, ion generation from ion sources is stochastic and, therefore, $\mathbf{x}$ varies on the time scale of the acquisition. Thus, each acquisition element of $\mathbf{y}$ with time index $t$ (corresponding to the time $t \Delta t$, where $\Delta t$ is the temporal width of the acquisition bin) records a unique population given by:

$$
y_{t}=\mathbf{s}_{t} \cdot \mathbf{x}_{t}+\varepsilon_{t}
$$

where $\mathbf{s}_{t}$ is the row $t$ of $\mathbf{S}$ and $\mathbf{x}_{t}$ is the population of ions that would arrive during $y_{t}$ in the absence of encoding. Fluctuations in $\mathbf{x}$ produce a variance in the recorded values of $y_{t}$. Deconvolution spreads this variance over all bins and causes signal-dependent noise to appear in all channels of the time-of-flight spectrum.

To understand the nature of this noise and to determine its significance, we have compared HT-TOFMS experimental data to probabilistic simulations and theory. These results provide an estimate of signaldependent baseline noise as a function of mass spectral features and acquisition parameters, and set the conditions for which Hadamard-multiplexed ion-counting TOFMS experiments are advantageous.

\section{Experiment and Simulation}

\section{Reagents}

The samples used for HT-TOFMS experiments were tetrabutylammonium acetate (TBA, Sigma Chemical, St. Louis, $\mathrm{MO}, \mathrm{Mw}=301.5 \mathrm{~g} \mathrm{~mol}^{-1}$ ), tetraethylammonium chloride (TEA, Sigma Chemical, St. Louis, MO, Mw = $165.7 \mathrm{~g} \mathrm{~mol}^{-1}$ ), and polypropylene glycol (PPG, narrow molecular-weight distribution centered about $450 \mathrm{~g}$ $\mathrm{mol}^{-1}$; Scientific Polymer Products, Ontario, NY). All solutions were $100 \mu \mathrm{M}$ in a 50:50 vol/vol mixture of high-purity water (18 $\left.\mathrm{M} \Omega \mathrm{cm}^{-1}\right)$ and methyl alcohol (Aldrich Chemical, Milwaukee, WI). Analytes were used as received without further purification.

\section{Electrospray Ionization HT-TOFMS}

Analyte solutions were driven through 50-micron i.d. fused-silica capillaries (Polymicro Technologies, Phoenix, AZ) at flow rates between 0.1 and $1 \mu \mathrm{L} / \mathrm{min}$ by application of a backpressure and were electrosprayed from the exit, which was sharpened and goldcoated [12]. The electrospray tip was held at voltages between 2500 and $3500 \mathrm{~V}$ and positioned roughly $5 \mathrm{~mm}$ from the grounded entrance orifice.

Figure 1 presents a schematic diagram of the HT-TOF mass spectrometer used in this work. A full description of this instrument can be found elsewhere [13]. Electrosprayed ions are collimated in an rf-only hexapole (2.9
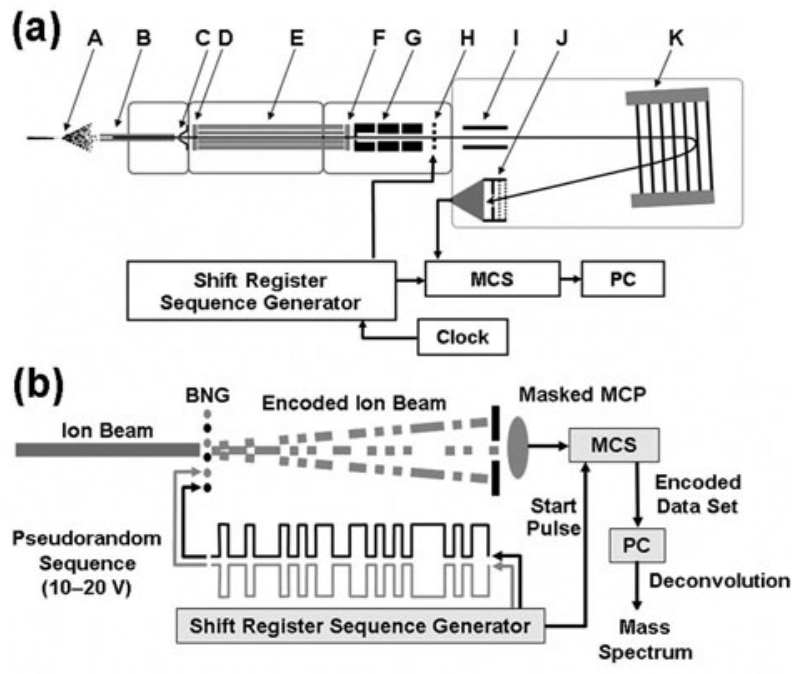

Figure 1. (a) Schematic of the HT-TOFMS experimental setup. A) Electrospray ion source, B) heated glass capillary, C) skimmer, D) focusing lens, E) hexapole, F) conductance limiting exit lens, G) Einzel lens, H) Bradbury-Nielson gate, I) x,y steering plates, J) masked MCP detector, and K) reflectron. (b) Schematic of the system for beam encoding and data acquisition. The shift-register pseudorandom sequence generator drives the two wires sets of the BNG with simplex-type encoding sequences with equal magnitude, opposite polarity bias voltages. The sequence generator sends start pulses to the MCS for synchronization. Recorded data are deconvoluted in the computer to obtain mass spectra.

MHz, 200 to $2500 \mathrm{~V}$, ABB Inc, Pittsburgh, PA) held at a pressure of $1 \times 10^{-4}$ torr before being accelerated to 1500 $\mathrm{eV}$ between the exit of the hexapole and the first element of the Einzel lens that follows the hexapole. Encoding sequences are applied to the Bradbury-Nielson gate, which is mounted just past the Einzel lens. The wires of the gate (20- $\mu \mathrm{m}$ gold-plated tungsten, $100-\mu \mathrm{m}$ spacing) float at the acceleration voltage of the instrument and are aligned such that beam deflection is along the vertical axis. High voltage and deflection bias voltages are supplied to the gate by a HT-TOFMS control system that was engineered in partnership with Predicant Biosciences (South San Francisco, CA). This PC-controlled system consists of a central control box that generates variable length, variable rate pseudorandom sequences, and vacuummounted driver circuitry that applies the acceleration voltage and the time-varying bias voltages to the BNG. For any applied encoding sequence, the temporal width of the encoding elements is the reciprocal of the sequence generator's clocking frequency. The total duration of one acquisition pass, which determines the mass range investigated, is the product of the element width and the sequence length.

Time zero of an ion's flight time is defined as the moment it crosses the plane of the BNG wires. The total flight path past the BNG is $2.2 \mathrm{~m}$. Ions are energy focused by a two-stage reflectron, which directs them toward the 40-mm multichannel plate detector (chevron configuration, Quantar Technology, Inc., Santa Cruz, CA). A mask having a horizontal opening ( $8 \mathrm{~mm}$ wide, 
$4 \mathrm{~mm}$ high) is mounted directly in front of the MCP. Focused ions are transmitted through this slit, while deflected ions are blocked by the mask and do not reach the detector.

The MCP output is amplified (10×, EG \& G Ortec model VT120, Oak Ridge, TN) and fed into a multichannel scaler (EG \& G Ortec Turbo MCS) for ion counting. The MCS is synchronized with the sequence by a TTL start pulse that is output at the start of every sequence. An acquisition pass has a total duration equal to that of the acquisition element multiplied by the length of the applied sequence. Data of successive passes are summed to improve SNR. In standard mode, ion counts are binned in acquisition elements with duration equal to that of the encoding elements. Experiments in this work utilized 50-, 100-, 200-, or 400-ns elements. The maximum ion count per acquisition element in each pass is determined by the input register size of the MCS and the acquisition rate. For 50-ns acquisition, the MCS can record up to 15 ion arrivals per element. At all other values it can record up to $10^{6}$ ion arrivals per acquisition element. Real-time deconvolution and data processing are performed with a program written in Delphi, which utilizes direct multiplication by the inverse-simplex matrix.

\section{Probabilistic Simulations}

The simulation routine, written in MATLAB (MathWorks, Natick, MA), constructs conventional TOF and HT-TOF mass spectra for conditions where the ion flux at the detector is varying during acquisition as a result of the stochastic nature of the ion beam. For each acquisition pass, $m$, the recorded data vector, $\mathbf{y}^{m}$, of length $N$, is built one element at a time based on probabilistic ion arrivals.

For species $i$ constituting the fraction $F_{i}$ of a beam with total ion counts per second TIC, the probability of an ion arrival within any acquisition bin of duration $\Delta t$ is calculated as:

$$
p_{i}=(T I C)(\Delta t)\left(F_{i}\right)
$$

Arrival events in this ion counting experiment are assumed to follow Poissonian statistics [14], and $p_{i}$ is input to the MATLAB Poisson random number generator (poissrnd) in order to produce the integer $x_{t, i}^{m}$, which represents the instantaneous population of species $i$ at time index $t$ of pass $m$. Ions are only recorded at time index $t$ if they are both present $x_{t, i}^{m}>0$ and allowed by the encoding sequence ( $\mathbf{s}_{t}$ for HT spectra and $\mathbf{i}_{t}$ for conventional TOF spectra, which is the row $t$ of $\mathbf{S}$ and $\mathbf{I}$, respectively). The simulation algorithm is optimized for speed such that multiplication steps involving the elements of species with $p_{i}=0$ are skipped. Dark counts and detector noise are assumed to be negligible, so the acquired data vector, $\mathbf{y}^{m}$, contains only ion counts.

In order to construct the vector $\mathbf{y}^{m}$, this process, from the generation of $x_{t}^{m}$ to the determination of $y_{t}^{m}$, is repeated for $t=1$ through $N$; and, $\mathbf{y}^{m}$ from successive passes are summed to produce the final acquisition vectors, $\mathbf{y}^{\mathrm{TOF}}$ and $\mathbf{y}^{\mathrm{HT}} \cdot \mathbf{y}^{\mathrm{HT}}$ is multiplied by the decoding matrix, $\mathbf{R}$, which is the inverse matrix $\mathbf{S}^{-1}$ scaled by $(N+1) / 2$ in order to remove the normalization factor of $2 /(N+1)$. This scaling is necessary because all ion counts in the raw data $\mathrm{y}^{\mathrm{HT}}$ result from real ions and the peak intensities after deconvolution should represent the actual number of measured ions.

Insufficient bias deflection voltages applied to the electrodes of the BNG and/or non-idealities in the shape of the applied electronic encoding sequence may produce incomplete deflection of the ion beam [3]. In this event, a fraction $\delta$ of the total ion current reaches the detector in both " 1 " and " 0 " states, and the encoded information is lost. This effect is simulated by using the same Poisson number generator with a reduced probability of $\delta p_{i}$ for sequence element 0 . As in experiment, deconvolution always assumes $\delta=0$. In practice, $\delta$ can be measured by comparing total ion counts with the sequence running, with the BNG fixed in its transmission state, and with the BNG fixed in its deflection state.

Mass spectra of TEA, TBA, and PPG were simulated using inputs (TIC, peak position, and relative peak intensities) derived from parallel HT-TOFMS experiments. Values of $p_{i}$ for all species were experimentally varied by adjusting the width of the acquisition bins $(\Delta t)$ and by adjusting source conditions in order to raise or lower total ion counts (TIC). All spectra were simulated for two values of $\delta$, one with the experimentally determined $\delta$ value and the other assuming perfect deflection $(\delta=0)$. The TEA and TBA spectra were simulated with exact peak shapes constituting several acquisition elements. To reduce computing time, each peak in the PPG spectrum was simulated as a single acquisition bin with intensity equal to the area of the corresponding experimental peak. All data are presented along the time-of-flight axis in order to demonstrate the equal distribution of baseline noise.

\section{Theory}

Several assumptions are made for calculating the intensity and the variance of HT-TOF and conventional TOF mass spectra. Detailed derivations for the key equations presented in this section can be found in the Appendix; only the results of the theoretical analysis are presented here.

\section{Assumptions}

1. The ion current is low, such that saturation effects from simultaneous arrivals of ions at the detector are negligible. Each data acquisition bin $\left(y_{t}^{m}\right)$ can record multiple ion arrivals and the maximum count allowed by hardware is effectively unlimited.

2. In order to record a spectrum, multiple acquisition passes are summed. All passes are independent of each 
other, and as a result, the means and the variances of multiple passes are additive.

3. If signal-independent dark current noise is present, its mean and variance are given by

$$
\left\langle\varepsilon_{i}^{m}\right\rangle=p_{d}, \quad\left\langle\left(\varepsilon_{i}^{m}\right)^{2}\right\rangle-\left\langle\varepsilon_{i}^{m}\right\rangle^{2}=\sigma_{d}^{2}
$$

where $p_{d}$ is the probability of observing dark current ions in an acquisition bin, $y_{t}^{m}$.

4. Discrete noise that originates from imperfections in the encoding (overshoot, ringing, etc) is negligible.

5. The number of ions detected in a single acquisition bin follows Poissonian statistics. By definition, the mean and the variance of the element $x_{t, i}^{m}$ for all $m$ and $t$ are thus:

$$
\left\langle x_{t, i}^{m}\right\rangle=p_{i}, \quad\left\langle\left(x_{t, i}^{m}\right)^{2}\right\rangle-\left\langle x_{t, i}^{m}\right\rangle^{2}=\sigma_{x_{i}}^{2}=p_{i}=\left\langle x_{t, i}^{m}\right\rangle
$$

6. Encoding having incomplete deflection can be described as a sum of encoded and uncorrelated components. The uncorrelated component has a reduced mean and variance

$$
\left\langle\delta x_{t, i}^{m}\right\rangle=\delta p_{i}, \quad\left\langle\left(\delta x_{t, i}^{m}\right)^{2}\right\rangle-\left\langle\delta x_{t, i}^{m}\right\rangle^{2}=\sigma_{\delta x_{i}}^{2}=\delta p_{i}=\left\langle\delta x_{t, i}^{m}\right\rangle
$$

where $\delta$ is the fraction of unmodulated ions $(0 \leq$ $\delta<1)$.

\section{Signal Intensities in HT-TOF and Conventional TOF Mass Spectra}

For an acquisition pass with index $m$, in a standard HT-TOFMS experiment having a total number of acquisition bins equal to the length of the encoding sequence, $N$, the data recorded at the detector is represented by $\mathbf{y}^{m}=\left[\begin{array}{llll}y_{1}^{m} & y_{2}^{m} & \cdots & y_{N}^{m}\end{array}\right]^{\mathrm{T}}$. The encoding process that produces this vector is represented by:

$$
y_{t}^{m}=\mathbf{s}_{t} \cdot \mathbf{x}_{t}^{m}+\left(\mathbf{j}-\mathbf{s}_{t}\right) \cdot \delta \mathbf{x}_{t}^{m}+\varepsilon_{t}^{m}
$$

where $\delta$ is the fraction of the total ion current that is unaffected by encoding, $\mathbf{s}_{t}$ is the $t$-th row of $\mathbf{S}$, and $\mathbf{j}$ is a row vector of length $N$ with all elements equal to 1. The total data $\mathbf{y}^{\mathrm{HT}}$, which is the sum of $\mathbf{y}^{m}$ for $M$ passes, is deconvoluted by software using the decoding matrix, $\mathbf{R}$, to obtain the mass spectrum, $\mathrm{z}^{\mathrm{HT}}=\left[\begin{array}{llll}z_{1} & z_{2} & \cdots & z_{N}\end{array}\right]^{\mathrm{T}}$.

$$
\mathbf{z}^{\mathrm{HT}}=\mathbf{R} \mathbf{y}^{\mathrm{HT}}=\left(\frac{N+1}{2} \mathbf{S}^{-1}\right) \sum_{m=1}^{M} \mathbf{y}^{m}
$$

The expectation value for the peak intensity of an ion species $i$ is given by

$$
\left\langle z_{i}^{\mathrm{HT}}\right\rangle=(1-\delta) \frac{N+1}{2} M p_{i}+\delta \sum_{k=1}^{N} M p_{k}+M p_{d}
$$

The HT-TOF signal intensity contains three terms:

$$
\begin{aligned}
\left\langle z_{i}^{\mathrm{HT}}\right\rangle_{\mathrm{S}} & =(1-\delta) \frac{N+1}{2} M p_{i} \\
\left\langle z_{i}^{\mathrm{HT}}\right\rangle_{0} & =\delta \sum_{k=1}^{N} M p_{k} \\
\left\langle z_{i}^{\mathrm{HT}}\right\rangle_{\mathrm{N}} & =M p_{d}
\end{aligned}
$$

They correspond to the signal intensity for the $i$ th peak (S), the intensity from unmodulated ions (0), and the dark current noise counts $(\mathrm{N})$, respectively.

In the equivalent conventional TOFMS experiment, single packets of duration $\Delta t$ are released into the mass analyzer with a period of $N \Delta t$. Thus, the duty cycle is $1 / N$. Here, $\mathbf{S}$ is replaced by the identity matrix, I, and the encoding is represented by:

$$
y_{t}^{m}=\mathbf{i}_{t} \cdot \mathbf{x}_{t}^{m}+\left(\mathbf{j}-\mathbf{i}_{t}\right) \cdot \delta \mathbf{x}_{t}^{m}+\varepsilon_{t}^{m}
$$

The TOF mass spectrum, $\mathbf{z}^{\mathrm{TOF}}$, is exactly equal to the recorded data, $\mathbf{y}^{\text {TOF}}$ :

$$
\mathbf{z}^{\mathrm{TOF}}=\mathbf{y}^{\mathrm{TOF}}=\sum_{m=1}^{M} \mathbf{y}^{m}
$$

The expectation value for the peak intensity of ion species $i$, having a flight time $t \Delta t$ corresponding to the element with time index $t$, is given by:

$$
\left\langle z_{i}^{\mathrm{TOF}}\right\rangle=(1-\delta) M p_{i}+\delta \sum_{k=1}^{N} M p_{k}+M p_{d}
$$

The expected intensity in conventional TOFMS has three terms with the same definitions as those of HT-TOFMS:

$$
\begin{aligned}
& \left\langle z_{i}^{\mathrm{TOF}}\right\rangle_{\mathrm{S}}=(1-\delta) M p_{i} \\
& \left\langle z_{i}^{\mathrm{TOF}}\right\rangle_{0}=\delta \sum_{k=1}^{N} M p_{k} \\
& \left\langle z_{i}^{\mathrm{TOF}}\right\rangle_{\mathrm{N}}=M p_{d}
\end{aligned}
$$

The intensity from unmodulated ions and the dark current noise are the same for both techniques. However, signal intensity for the $i$ th peak in an HT-TOF mass spectrum is larger by a factor of $(N+1) / 2$, owing to the fact that approximately half of the elements in the $N$-element simplex-type encoding sequence transmit ions.

\section{The Variance for HT-TOF and Conventional TOF}

Whereas conventional TOFMS measures ions species independently, any given acquisition bin, $y_{t}^{m}$, in an HT-TOFMS experiment represents the simultaneous measurement of $(N+1) / 2$ ion species, with allowed identities defined by the encoding sequence. The inten- 
sity and the uncertainty of $y_{t}^{m}$ derive from the signals of all allowed species. The variance of any measurement is described by:

$$
\operatorname{Var}\left(y_{t}^{\mathrm{HT}}\right)=\sum_{k=1}^{N}(\mathbf{S})_{t k} M p_{k}+\sum_{k=1}^{N}\left(1-(\mathbf{s})_{t k}\right) M \delta p_{k}+M \sigma_{d}^{2}
$$

The decoding matrix $\mathbf{R}$ rearranges all recorded ion counts according to their identity, producing the vector $\mathbf{z}^{\mathrm{HT}}$. Just as the intensity of any element $z_{i}^{\mathrm{HT}}$ depends on the recorded values of all $y_{t}^{\mathrm{HT}}$, the variance of each $z_{i}^{\mathrm{HT}}$ is the sum of the variances of all $y_{t}^{\mathrm{HT}}$. That is, all elements of the deconvoluted spectrum have the same variance, which is the sum of the variances of all acquisition bins:

$$
\operatorname{Var}\left(z_{i}^{\mathrm{HT}}\right)=\sum_{t=1}^{N} \operatorname{Var}\left(y_{t}^{\mathrm{HT}}\right)
$$

Substituting yields the expanded expression for the variance of $z_{i}^{\mathrm{HT}}$ :

$$
\begin{aligned}
\operatorname{Var}\left(z_{i}^{\mathrm{HT}}\right)= & (1-\delta) \frac{N+1}{2} \sum_{k=1}^{N} M p_{k}+\delta N \sum_{k=1}^{N} M p_{k} \\
& +N M \sigma_{d}^{2}
\end{aligned}
$$

which has three terms, originating from the signal intensity fluctuation (S), unmodulated ions (0), and dark current noise $(\mathrm{N})$, respectively.

$$
\begin{aligned}
& \operatorname{Var}\left(z_{i}^{\mathrm{HT}}\right)_{\mathrm{S}}=(1-\delta) \frac{N+1}{2} \sum_{k=1}^{N} M p_{k} \\
& \operatorname{Var}\left(z_{i}^{\mathrm{HT}}\right)_{0}=\delta N \sum_{k=1}^{N} M p_{k} \\
& \operatorname{Var}\left(z_{i}^{\mathrm{HT}}\right)_{\mathrm{N}}=N M \sigma_{d}^{2}
\end{aligned}
$$

For the conventional case, only ions of species $i$ are allowed to arrive within $y_{t}^{\mathrm{TOF}}$ and the variance of the value of any species is no longer dependent on the signals of other species.

$$
\operatorname{Var}\left(z_{i}^{\mathrm{TOF}}\right)=(1-\delta) M p_{i}+\delta \sum_{k=1}^{N} M p_{k}+M \sigma_{d}^{2}
$$

Again, this expression can be decomposed into three components:

$$
\begin{aligned}
& \operatorname{Var}\left(z_{i}^{\mathrm{TOF}}\right)_{\mathrm{S}}=(1-\delta) M p_{i} \\
& \operatorname{Var}\left(z_{i}^{\mathrm{TOF}}\right)_{0}=\delta \sum_{k=1}^{N} M p_{k} \\
& \operatorname{Var}\left(z_{i}^{\mathrm{TOF}}\right)_{\mathrm{N}}=M \sigma_{d}^{2}
\end{aligned}
$$

All three components have smaller magnitude than the corresponding Hadamard expressions.

\section{Total Ions in a Spectrum}

For comparison with experimental data, it is convenient to relate these equations with spectral quantities that can be measured experimentally. $\Psi_{1}$ and $\Psi_{0}$ are the total numbers of ion counts during the spectral acquisition time $(N M \Delta t)$ when the BNG is fixed at states 1 and 0 , respectively. They are given by:

$$
\begin{aligned}
& \Psi_{1} \equiv N \sum_{i=1}^{N} M p_{i} \\
& \Psi_{0} \equiv \delta N \sum_{i=1}^{N} M p_{i}
\end{aligned}
$$

Thus, the proportion of unmodulated ions, $\delta$, can be estimated experimentally by their ratio, $\Psi_{0} / \Psi_{1}$.

The total number of ions in an HT mass spectrum, $\Psi_{\mathrm{S}}$, can be expressed as:

$$
\Psi_{\mathrm{S}} \equiv \sum_{i=1}^{N} z_{i}^{\mathrm{HT}}=f_{\mathrm{S}}\left(\Psi_{1}-\Psi_{0}\right)+\Psi_{0} \approx(1+\delta) f_{\mathrm{S}} \Psi_{1}
$$

where $f_{\mathrm{S}}$ represents the $50 \%$ duty cycle of the simplex encoding sequences used in HT-TOFMS.

$$
f_{\mathrm{S}}=\frac{N+1}{2 N} \approx 0.5
$$

The subscript $\mathbf{S}$ indicates the encoding following the $N$ $\times N$ simplex matrix $\mathbf{S}$. Likewise, $\Psi_{\mathbf{I}}$ is the total number of ions that would be recorded in an equivalent amount of time with conventional, identity-type encoding.

$$
\Psi_{\mathrm{I}} \equiv \sum_{i=1}^{N} z_{i}^{\mathrm{TOF}}=f_{\mathrm{I}}\left(\Psi_{1}-\Psi_{0}\right)+\Psi_{0} \approx f_{\mathrm{I}} \Psi_{1}
$$

where $f_{\mathbf{I}}$ describes the duty cycle of the identity-type encoding in conventional TOFMS.

$$
f_{\mathrm{I}}=\frac{1}{N}
$$

Experimentally, $\Psi_{\mathrm{S}}$ and $\Psi_{\mathrm{I}}$ are each obtained by summing the counts in all bins of the mass spectrum, whereas $\Psi_{1}$ and $\Psi_{0}$ must be measured separately. Thus, $\Psi_{\mathrm{S}}$ and $\Psi_{\mathrm{I}}$ are more convenient quantities to relate to the peak intensity and the variance.

For ions arriving with Poissonian statistics, eqs 23 and 27 for the variance of a bin, $z_{i}$, in HT-TOF and conventional TOF mass spectra can be expressed in terms of $\Psi_{1}$, duty cycles, and the fractional abundance of species $i, F_{i}$.

$$
\begin{aligned}
& \operatorname{Var}\left(z_{i}^{\mathrm{HT}}\right)=\left[(1-\delta) f_{\mathrm{S}}+\delta\right] \Psi_{1} \approx(1+\delta) f_{\mathrm{S}} \Psi_{1} \approx \Psi_{\mathrm{S}} \\
& \operatorname{Var}\left(z_{i}^{\mathrm{TOF}}\right)=\left[(1-\delta) F_{i}+\delta\right] f_{\mathrm{I}} \Psi_{1} \approx F_{i} f_{\mathrm{I}} \Psi_{1} \approx F_{i} \Psi_{\mathrm{I}}
\end{aligned}
$$

The variance of all bins in an HT-TOF mass spectrum is 
constant and depends only on $\Psi_{1}$. A plot of the variance of any bin versus $f_{\mathrm{S}} \Psi_{\mathbf{S}}$ should give a line with a slope of $1+\delta$. In contrast, the variance of species $i$ in a conventional TOF mass spectrum depends on only its abundance and is independent of all other species. Thus, the variance for the peak-free regions of the baseline should be almost zero.

From an examination of eq 11 for the peak intensity in HT-TOFMS, it is evident that the factor $(N+1) / 2$ causes the first term to dominate. Substituting $\Psi_{1}$ and $F_{i}$, the intensity of peak $i$ in an HT-TOF mass spectrum can be reduced to:

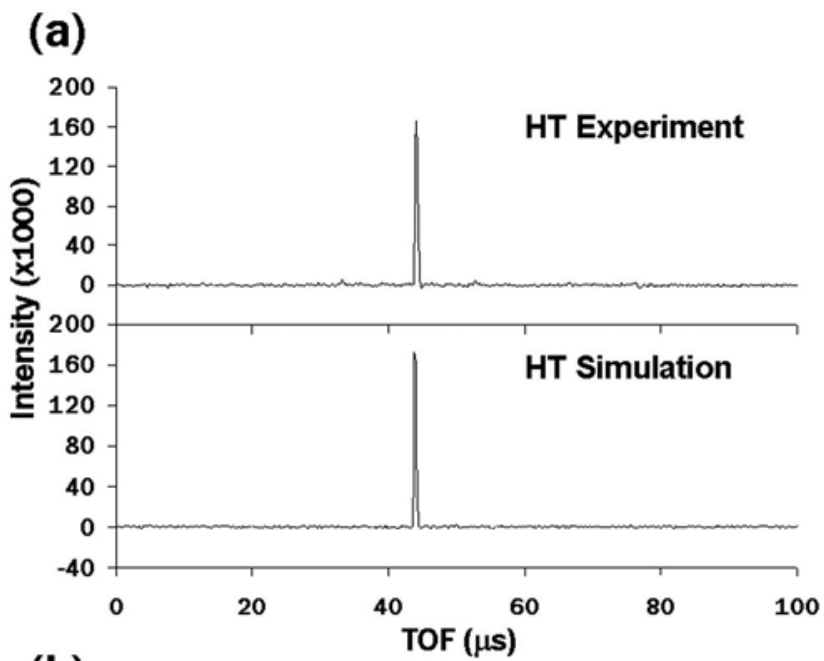

(b)

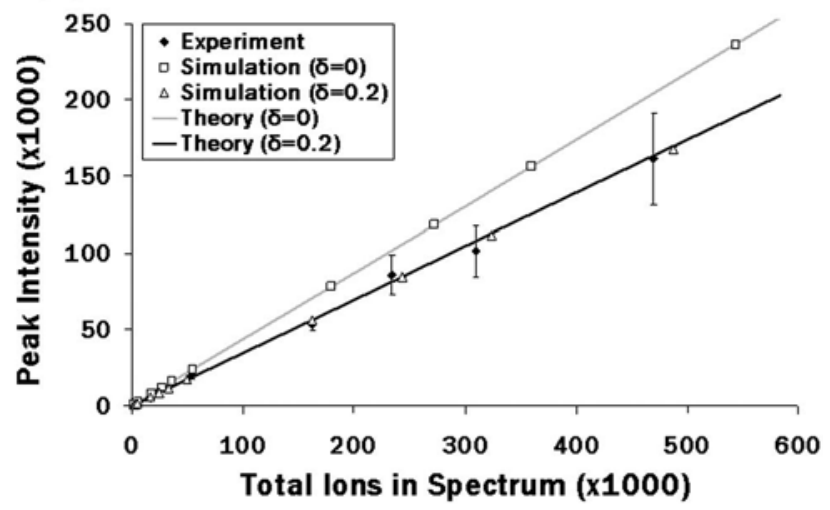

(c)

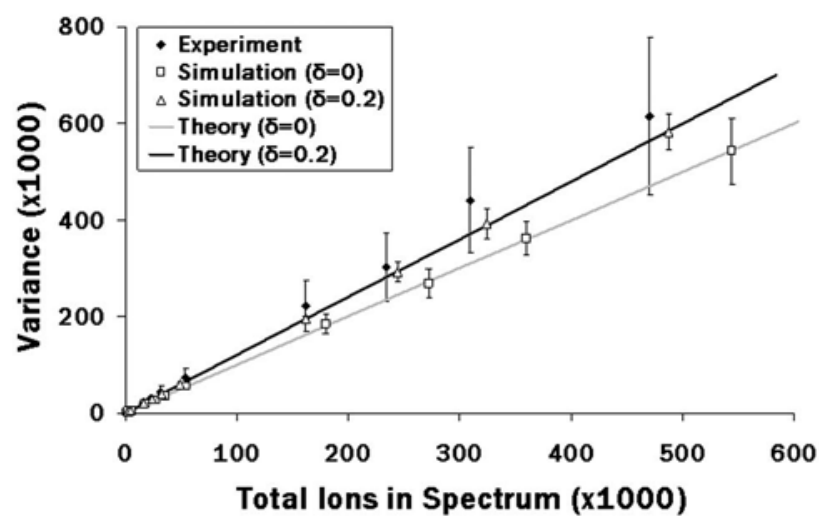

$$
\left\langle z_{i}^{\mathrm{HT}}\right\rangle \approx(1-\delta) F_{i} f_{\mathrm{S}} \Psi_{1} \approx \frac{1-\delta}{1+\delta} F_{i} \Psi_{\mathrm{S}}
$$

and eq 17 for conventional TOFMS can be simplified to give:

$$
\left\langle z_{i}^{\mathrm{TOF}}\right\rangle \approx\left[(1-\delta) F_{i}+\delta\right] f_{\mathrm{I}} \Psi_{1} \approx(1-\delta) F_{i} \Psi_{\mathrm{I}}
$$

For both, the height of any peak is proportional to the total ions in the spectrum and the fractional abundance of the species it represents. But, the HT-TOFMS peak is larger by the ratio of duty cycles $\left(f_{\mathbf{S}} / f_{\mathbf{I}}\right)$, which equals $(N+1) / 2$. A plot of HT-TOF mass spectral peak intensity as a function of $f_{\mathbf{S}} \Psi_{1}$ should give a line having a slope proportional to $(1-\delta)$.

\section{Results and Discussion}

\section{Origin of Noise}

For conditions where spectral noise is dominated by stochastic ion flux obeying Poissonian statistics, the variance of the recorded value of any bin in an HT-TOF mass spectrum will equal the total number of ions in the spectrum $\Psi_{\mathrm{S}}$. To test the validity of the expressions in the previous section and the assumptions about ion statistics, HT-TOF mass spectra of the salt tetraethylammonium chloride were collected and simulated at different ion currents, acquisition rates, and numbers of summed passes. Figure 2a displays examples of experimental and simulated spectra, which demonstrate general agreement in appearance.

Figure $2 \mathrm{~b}$ plots experimental tetraethylammonium ion $\left(\mathrm{TEA}^{+}\right)$peak heights. $\Psi_{\mathrm{S}}$ was varied by adjusting source conditions to limit current and by varying the numbers of passes summed. Data are plotted with corresponding results from simulations and lines predicted by theory (eq 39), for conditions where encoding is ideal $(\delta=0)$ and for the experimentally determined value $\delta=0.20$. To study more clearly the effects of

Figure 2. Experimental and simulated HT-TOF mass spectra of tetraethylammonium $\left(\mathrm{TEA}^{+}\right)$chloride. (a) $\mathrm{TEA}^{+}$spectra, which were collected with approximate TIC of $1 \times 10^{5}$ ions/s, 511-element sequences, and 200-ns acquisition bins (5 MHz) and summed for $1 \times 10^{5}$ passes. (b) Peak height versus total ions in spectrum $\left(f_{\mathbf{s}} \Psi_{1}\right) . \Psi_{1}$ was varied by adjusting source conditions to limit current and by varying the numbers of passes summed. TEA ${ }^{+}$spectra were collected and simulated for approximate TICs of $7.0 \times 10^{4}$ and $1.1 \times 10^{5}$ ions/s encoding with a sequence containing 511 200-ns bins. Experiments and simulations were repeated 16 times at 500, 1000, 5000, $1 \times$ $10^{4}, 5 \times 10^{4}$, and $100 \times 10^{5}$ passes. The simulated peak shape was based on experiment. Data points represent the intensity of the most intense bin in the spectrum $\left(F_{i}=0.43\right)$, with error bars corresponding to 1 standard deviation. All simulations were run for the ideal deflection, $\delta=0$, and the experimentally achieved, $\delta=0.2$. Theoretical lines are based on eq 39. (c) Variance of $\mathrm{TEA}^{+}$spectra. Values were calculated as the variance of the bins in the peak-free region between 0 and $10 \mu \mathrm{s}$. All simulations were run for $\delta=0$ and $\delta=0.2$. Theoretical lines are based on eq 37. 
unmodulated ions, experimental conditions were chosen to have a higher $\delta$ value than its typical value, which is less than 0.10 . The slopes of all three data sets at $\delta=0.20$ are identical within error bars. There are slight differences between the recorded values for simulations and experiment along the total ions in spectrum $\left(f_{\mathrm{S}} \Psi_{1}\right)$ axis, which becomes evident at high values. This behavior is likely caused by discrepancies in the input values of TIC and the $F_{i}$ value of the most intense bin of the $\mathrm{TEA}^{+}$peak.

Figure 2c plots the variance for these data, which was calculated as the variance of the values of elements in a peak-free portion of the spectra. Because the HT method spreads random noise evenly throughout spectra, this value represents the variances in the values of all elements. For the simulated case where deflection is ideal, the baseline elements have intensities that integrate to 0 and the area of the $\mathrm{TEA}^{+}$peak equals $\Psi_{\mathrm{S}}$. Incomplete deflection yields uncorrelated ion counts that are evenly spread across the entire spectrum and reduces the peak height by a factor of $(1-\delta)$ (see eq 39). This effect raises the recorded variance (see eq 37 ).

Agreement between simulation and experiment for the variance is always within error bars, but experiment displays positive deviations that grow more significantly at high $\Psi_{1}$. This behavior is believed to be the manifestation of slow fluctuations in the electrospray current that do not follow Poissonian statistics. Work in Hadamard optical spectroscopy has shown that the observed variance can depend on both photon shot noise, which is Poissonian, and $1 / \mathrm{f}$ type source fluctuations [9, 15]. Standard deviation of the latter grows linearly with intensity. Spectral analysis of time traces of total ion current from our electrospray source shows 1 /f noise below $30 \mathrm{~Hz}$ [16], which is consistent with the low-frequency pulsation reported by Wei et al. [17]. The resultant deviation from Poissonian statistics only becomes appreciable when an unusually high number of passes are summed. Thus, $1 / \mathrm{f}$ noise is not significant for typical HT-TOFMS acquisition rates.

The same dependences of peak height and variance on $\Psi_{\mathrm{S}}$ are demonstrated in Figure 3, for the spectrum of polypropylene glycol (PPG) containing six primary peaks (shown in Figure 3a). Simulations and theory are shown for $\delta$ equal to 0 and 0.15 .

Figure $3 \mathrm{~b}$ plots the area of the peak at $87.2 \mu \mathrm{s}$ having $F_{i}$ of 0.36 ; Figure $3 \mathrm{c}$ plots the variance as calculated in the region between 0 and $10 \mu \mathrm{s}$. Again, we see deviations in the variance at high $\Psi_{1}$, but these data strongly suggest that the variance of all bins is a function of the $\Psi_{1}$ and can be estimated assuming Poissonian ion statistics (eq 37).

\section{Peak Height Precision}

Multiplexing has the advantage of increasing the intensity of recorded peak heights per unit time. For experiments with signal-dependent variance, all peaks are measured above a baseline noise having a magnitude
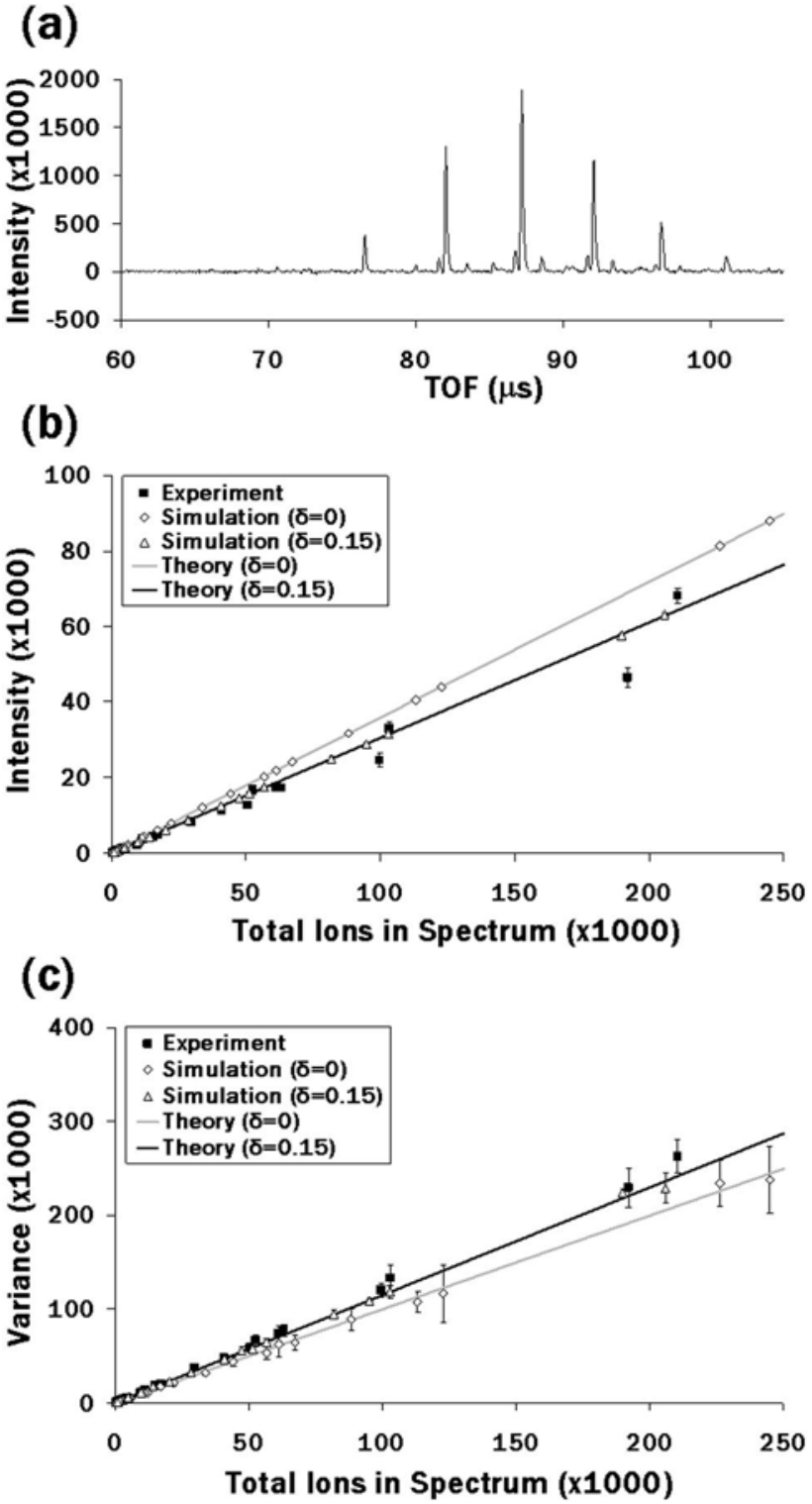

Figure 3. Experimental and simulated HT-TOFMS data for polypropyleneglycol (PPG). (a) Example of an HT-TOF mass spectrum of PPG collected using a 4095-element, 20-MHz encoding sequence. TIC in this experiment was $1.1 \times 10^{5}$ ions/s. The six dominant peaks were included in the simulations. (b) PPG peak area vs. total ions in spectrum $\left(f_{\mathrm{S}} \Psi_{1}\right)$. Spectra were collected with acquisition length/rate of 511/2.5, 1023/5.0, 2047/10, and 4095 element $/ 20 \mathrm{MHz}$, and approximate TIC of $1.2 \times 10^{5}, 3.3 \times 10^{4}, 4.3 \times 10^{4}$, and $1.1 \times 10^{5}$ ions/s, respectively. Data were summed for 100, 500, $1000,5000,1 \times 10^{4}$, and $2 \times 10^{4}$ passes. Simulations were run with each of the six peaks represented by a single bin with intensity equal to the area of the peak. Data points correspond to the area of the most intense peak at $87.2 \mu \mathrm{s}\left(F_{i}=0.36\right)$. All simulations were run for the ideal deflection, $\delta=0$, and the experimentally achieved, $\delta=0.15$. Theoretical lines are based on eq 39. (c) Variance of baseline noise as a function of $f_{\mathrm{S}} \Psi_{\mathrm{S}}$ under the same conditions as in Figure $3 \mathrm{~b}$. Theoretical lines are based on eq 37.

that depends on the populations of all spectral components. This behavior is illustrated in Figure 4, where the PPG spectrum has been simulated with ion species removed in order of intensity. Each spectrum contains 


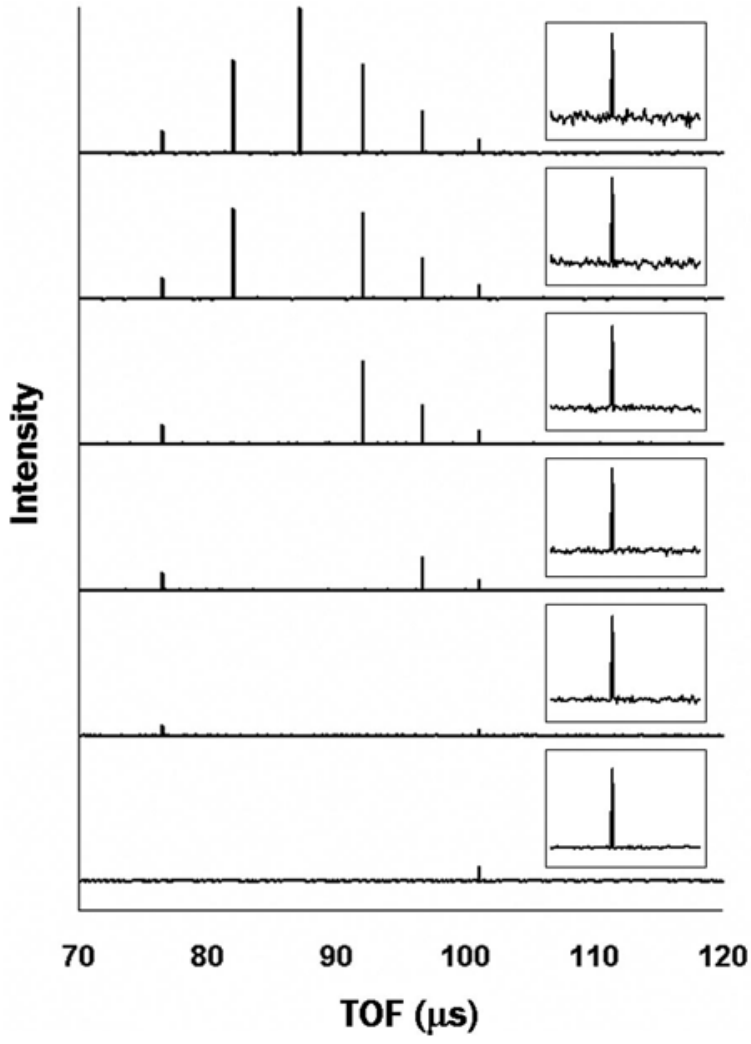

Figure 4. Simulated PPG spectra with dominant peaks removed. 4095-element, 20-MHz HT-TOF mass spectra of PPG were summed for $5 \times 10^{4}$ passes. The first spectrum contains all six peaks and has TIC of $1.1 \times 10^{5}$ ion/s. Peaks and their ions counts were sequentially removed, beginning with the most intense. The inset in each spectrum shows the smallest peak with $F_{i}=0.035$, $0.055,0.085,0.182,0.398$, and 1 , from top to bottom.

an inset showing the smallest peak (at $101.1 \mu \mathrm{s}$ ) and the surrounding baseline. While the intensity of this peak never changes, its fractional population $\left(F_{i}=\right.$ $\left.p_{i} / \sum_{k=1}^{N} p_{k}\right)$ increases from 0.035 to 1 because other peaks have been removed. The simultaneous reduction in $\Psi_{\mathrm{s}}$ causes the standard deviation of the noise $\sqrt{\operatorname{Var}\left(z_{i}^{\mathrm{HT}}\right)}$ to be reduced by a factor of 0.18 .

We define SNR as the ratio of signal intensity to the standard deviation of the baseline,

$$
\mathrm{SNR}_{i}=\frac{\left\langle z_{i}\right\rangle_{\mathrm{S}}}{\sqrt{\operatorname{Var}\left(z_{b}\right)_{\mathrm{S}}+\operatorname{Var}\left(z_{b}\right)_{\mathrm{N}}}}
$$

where the index $i$ refers to the acquisition element containing the peak of interest and the index $b$ is any acquisition element that does not correspond to any ion species present in the beam. Under conditions where the baseline noise is dominated by detector noise, SNRs for both experiments are given by:

$$
\mathrm{SNR}_{i}^{\mathrm{HT}}=\frac{\left\langle z_{i}^{\mathrm{HT}}\right\rangle_{\mathrm{S}}}{\sqrt{\operatorname{Var}\left(z_{b}^{\mathrm{HT}}\right)_{\mathrm{N}}}}=\frac{N+1}{2 \sqrt{N}} \sqrt{M} \frac{p_{i}}{\sigma_{d}}
$$

$$
\mathrm{SNR}_{i}^{\mathrm{TOF}}=\frac{\left\langle z_{i}^{\mathrm{TOF}}\right\rangle_{\mathrm{S}}}{\sqrt{\operatorname{Var}\left(z_{b}^{\mathrm{TOF}}\right)_{\mathrm{N}}}}=\sqrt{M} \frac{p_{i}}{\sigma_{d}}
$$

These equations lead to the often-stated multiplexing advantage of $\sqrt{N} / 2$ (eq 1), but this conclusion is not appropriate for HT-TOFMS in conditions for which the detector noise is negligible and the ion shot noise is the dominant contributor to the baseline. When $\delta$ equals 0 and there are no dark counts, the baseline of the conventional experiment will have no appreciable intensity, and eq 43 would imply a nearly infinite value for $\mathrm{SNR}^{\mathrm{TOF}}$. Therefore, a more suitable measure of performance is peak height precision (PHP), which is the mean recorded peak height divided by the uncertainty in its measurement. For conditions dominated by Poissonian shot noise:

$$
\begin{aligned}
\operatorname{PHP}_{i}^{\mathrm{HT}} & =\frac{\left\langle z_{i}^{\mathrm{HT}}\right\rangle_{\mathrm{S}}}{\sqrt{\operatorname{Var}\left(z_{i}^{\mathrm{HT}}\right)_{\mathrm{S}}}}=\sqrt{\frac{N+1}{2}} \sqrt{F_{i} M p_{i}} \\
& \approx F_{i} \sqrt{f_{\mathrm{s}} \Psi_{1}}
\end{aligned}
$$

and

$$
\mathrm{PHP}_{i}^{\mathrm{TOF}}=\frac{\left\langle z_{i}^{\mathrm{TOF}}\right\rangle_{\mathrm{S}}}{\sqrt{\operatorname{Var}\left(z_{i}^{\mathrm{TOF}}\right)_{\mathrm{S}}}}=\sqrt{M p_{i}} \approx \sqrt{F_{i} \Psi_{\mathrm{I}} \Psi_{1}}
$$

Thus, the ratio of the peak height precision for HTTOFMS to that of an equivalent conventional TOFMS measurement is given by:

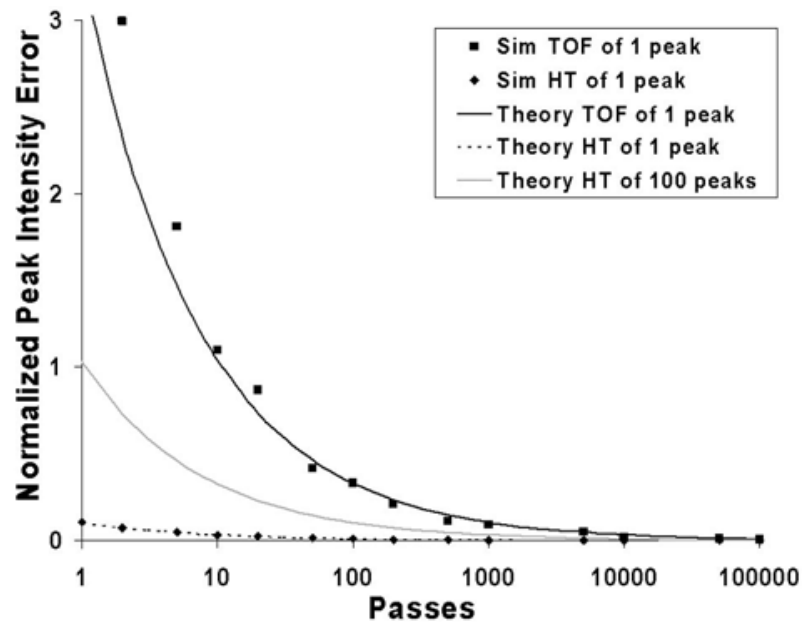

Figure 5. Normalized peak intensity error for simulated conventional TOF and HT-TOF mass spectra. 2047-Element, 10-MHz spectra containing a peak in a single bin were simulated by summing varied numbers of passes for conventional and HTTOFMS conditions. All simulations were run nine times. Data points represent the standard deviation of the peak height divided by the mean recorded value $(1 / \mathrm{PHP})$. Theoretical lines are shown for spectra containing the single peak and 100 equal peaks, collected under identical acquisition conditions. 

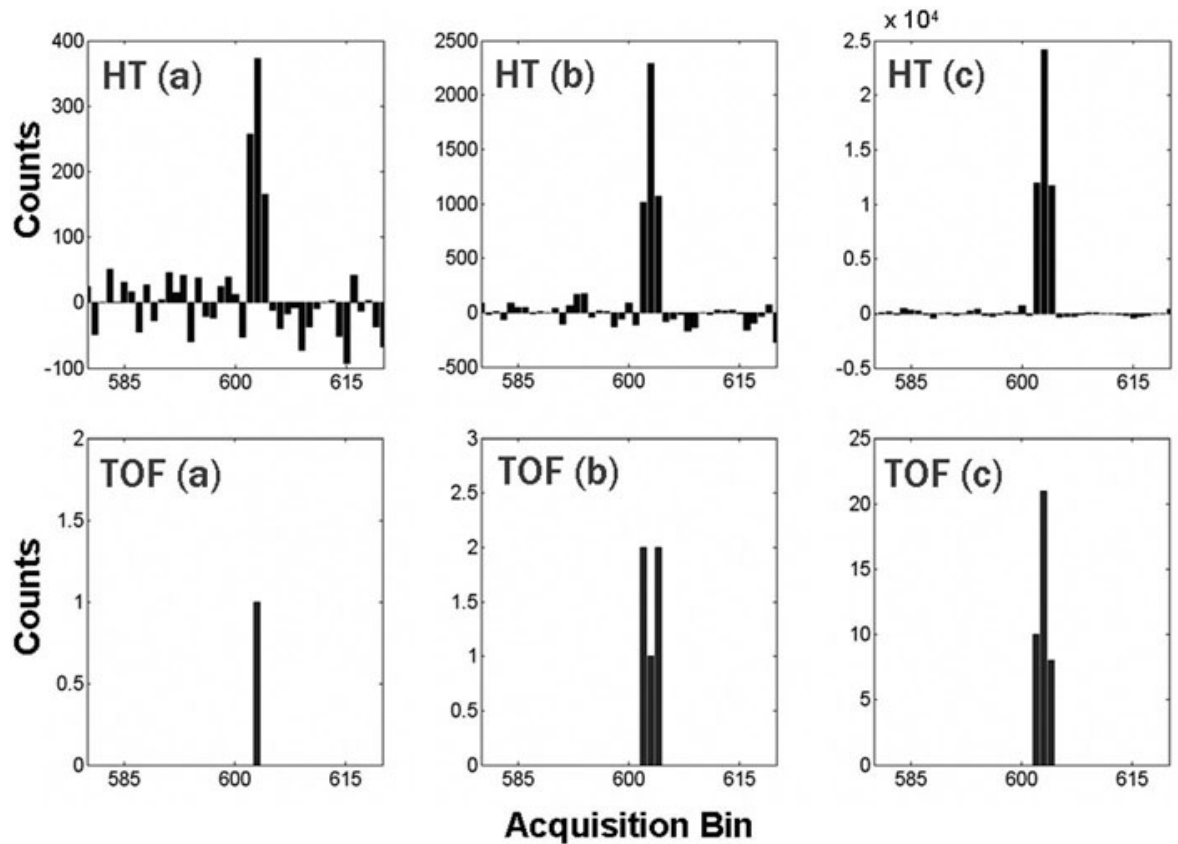

Figure 6. Simulated conventional TOF and HT-TOF mass spectra of tetrabutylammonium $\left(\mathrm{TBA}^{+}\right)$ acetate at fast acquisition rates. All simulations were run with a $10-\mathrm{MHz}, 2047$-element encoding sequence and a TIC of $9.25 \times 10^{5}$ ions $/ \mathrm{s}$. The $\mathrm{TBA}^{+}$peak constitutes three adjacent elements with $F_{i}$ of $0.25,0.5$, and 025 , respectively. (a) 10 passes, 500 spectra/s; (b) 100 passes, 50 spectra/s; (c) 500 passes, $10 \mathrm{spectra} / \mathrm{s}$.
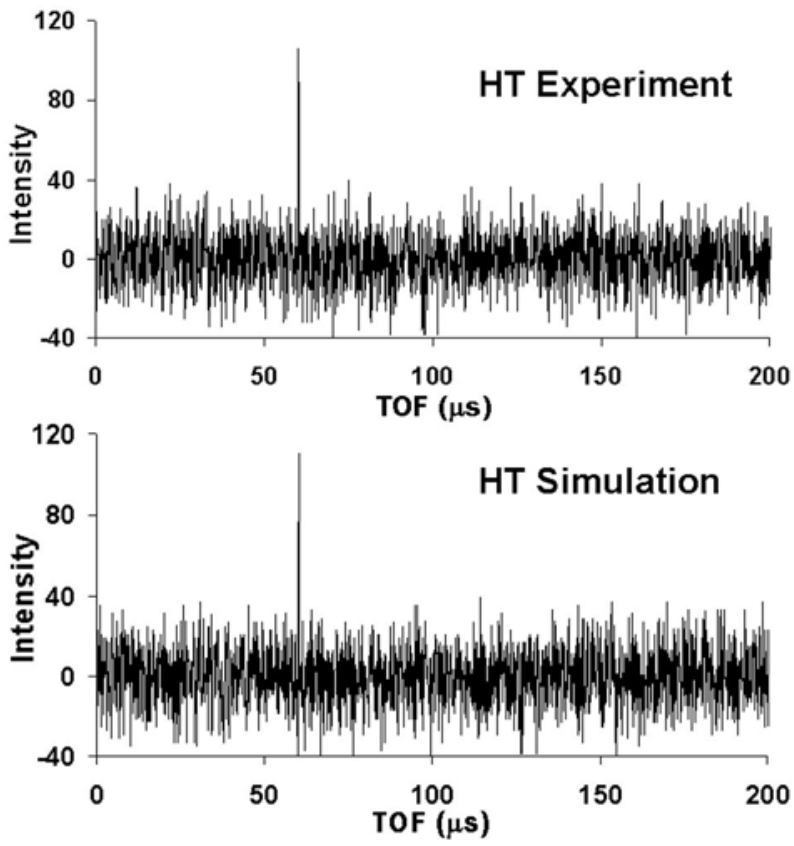

Figure 7. Experimental and simulated HT-TOF mass spectra of $\mathrm{TBA}^{+}$at an acquisition rate of 2500 spectra/s. Experimental conditions were identical to those described in Figure 6. These spectra were collected by summing 2 passes, corresponding to a spectral acquisition rate of $2500 \mathrm{spectra} / \mathrm{s}$. At this acquisition rate, the equivalent conventional experiment would be expected to record an average 0.185 ions/spectrum. In this spectrum, the HT-TOF instrument has recorded 168 ions.

$$
\frac{\mathrm{PHP}_{i}^{\mathrm{HT}}}{\mathrm{PHP}_{i}^{\mathrm{TOF}}}=\sqrt{\frac{N}{2} F_{i}}=\sqrt{\frac{f_{\mathrm{s}}}{f_{\mathrm{I}}}}
$$

We regard eq 46 as the key result of this work. This treatment, which includes the effects of duty cycle and signal-dependent noise, can be extended to compare HT-TOF with other TOF experiments beyond those using identity-type encoding. This is accomplished by replacing $f_{\mathrm{I}}$ in eq 46 with the experimental duty cycle for species $i$. Likewise, eq 46 can be used to describe two-channel HT-TOFMS [3] by replacing the onechannel duty cycle $f_{\mathbf{S}}$ with the sum of the duty cycles of the two detection channels.

For the simple case of a spectrum containing only one peak, $F_{i}$ is unity, and HT multiplexing improves peak precision by a factor of $\sqrt{N / 2}$, independent of its intensity. This behavior is demonstrated in Figure 5, which plots the inverse of $\mathrm{PHP}^{\mathrm{HT}}$ and $\mathrm{PHP}^{\mathrm{TOF}}$ for mass spectra having one peak and $N=2047$. This inverse value represents the normalized error in the reported peak height. Data points are the results of simulation, and the lines are the values predicted by eqs 44 and 45 . Figure 5 also contains the calculated result for an HT-TOF mass spectrum containing 100 identical peaks $\left(F_{i}=0.01\right)$, which begins to approach the conventional precision. According to eq 46, multiplexing will be disadvantageous for any peak with $F_{i}$ less than $2 / N$, where $N$ is typically $2^{11}-1=2047$. Thus, HT-TOFMS is advantageous compared to conventional TOF whenever it is required to measure the peak heights of 
species present in the ion beam at percentages greater than $0.1 \%$. Conversely, species present in percentages less than $0.1 \%$ are not determined with advantage using HT-TOFMS. Clearly, this result suggests that mixtures having species of interest that vary in concentration by more than a factor of 1000 are not well suited to determination by this technique. To maximize the advantage of HT-TOFMS, the method should be combined with separations schemes so that the abundance of the ion of interest is not diluted by the presence of other unwanted ions. Further, because $f_{\mathbf{S}}$ is constant whereas $f_{\mathbf{I}}$ depends on the mass range and/or mass resolution, the technique should achieve higher sensitivity in experiments where the duty cycle of other TOF experiments are low. Although ion storing techniques can achieve almost $100 \%$ duty cycle, they do not have real-time detection capability. A promising alternative is orthogonal acceleration time-of-flight mass spectrometry [18], but some trade-off must be accepted between mass range covered and sample utilization efficiency.

\section{Acquisition Rate}

When is it possible to exploit the peak height precision advantage of HT-TOFMS? One answer is when recording transient species, as is shown in what follows. For typical ion beam currents, very small numbers of ions will be released per TOF start pulse. Conventional ion counting experiments, which release only one pulse per pass, must sum multiple passes in order to determine precise peak heights and relative species populations. HT-TOFMS, on the other hand, releases and records thousands of sampling start pulses per pass. Figure 6 displays simulated HT-TOF and conventional TOF mass spectra at acquisition rates of 500, 100, and 10 spectra per second for a tetrabutylammonium $\left(\mathrm{TBA}^{+}\right)$ acetate peak that is three bins wide. The peak heights in HT-TOF mass spectra are, on average, three orders of magnitude greater than those of the conventional TOF. Further, Hadamard multiplexing allows true peak shapes (relative peak heights) to be recorded at rates where the conventional experiment detects only a few ions. This advantage of HT-TOFMS becomes more pronounced as the acquisition rate increases.

As a demonstration of these capabilities, Figure 7 shows experimental and simulated mass spectra of $\mathrm{TBA}^{+}$ under the same conditions as Figure 6 but collected at 2500 spectra per second (two 2047-element, 10-MHz passes). The experiment recorded 168 ions in $400 \mu$ s, while the equivalent conventional experiment with these ion currents and periodic pulsing would detect an average of 0.185 ions in the same time. This great difference suggests that HT-TOFMS is capable of monitoring transient processes on timescales where precision limits the use of equivalent conventional methods.

We conclude that under favorable conditions, the HT-TOFMS technique can be employed with a remarkable gain in the precision of the recorded peak heights of species appearing in the mass spectrum. This advan- tage becomes accentuated when the total ion current is low. This conclusion is a general one but requires that the species of interest be present at levels typically greater than $0.1 \%$ of the total ion beam current.

\section{Acknowledgments}

OT thanks the Deutsche Forschungsgemeinschaft (DFG) for an Emmy Noether-Fellowship (TR 542/1-1/2). This work was supported by the US Air Force Office of Scientific Research (AFOSR grant FA9550-04-1-0076).

\section{Appendix}

We present here the derivations of the key equations used in the text.

\section{A. Properties of a Simplex Matrix}

The simplex matrix $\mathbf{S}$, used in HT-TOFMS, is a rightcirculant matrix where the first column is the encoding sequence and the consecutive columns are copies of the first column shifted down cyclically. Therefore, $\mathbf{S}$ contains values of 0 and 1 only.

An $N \times N$ simplex matrix, $\mathbf{S}$, has the following properties [6].

$$
\begin{aligned}
& \mathbf{S}^{\mathrm{T}} \mathbf{S}=\mathbf{S S}^{\mathrm{T}}=\frac{N+1}{4}(\mathbf{I}+\mathbf{J}) \\
& \mathbf{S} \mathbf{J}=\mathbf{J S}=\frac{N+1}{2} \mathbf{J} \\
& \mathbf{S}^{-1}=\frac{2}{N+1}(2 \mathbf{S}-\mathbf{J}) \\
& \mathbf{S}^{-1} \mathbf{J}=\frac{2}{N+1} \mathbf{J}
\end{aligned}
$$

Here, $\mathbf{S}^{\mathrm{T}}$ refers to the transpose of $\mathbf{S}, \mathbf{S}^{-1}$ is the inverse of $\mathbf{S}, \mathbf{I}$ is the $N \times N$ identity matrix, and $\mathbf{J}$ is an $N \times N$ matrix where all elements are equal to 1.

\section{B. Equations for the Signal Intensity}

(a) HT-TOF (eq 11). In an HT-TOFMS experiment, ions that arrive at the detector are counted and summed for multiple passes by the multi-channel scaler (MCS). For a single acquisition pass with pass index $m$, the number of ions detected in an acquisition bin at time index $t, y_{t}^{m}$ is related to the encoding process by:

$$
y_{t}^{m}=\mathbf{s}_{t} \cdot \mathbf{x}_{t}^{m}+\left(\mathbf{j}-\mathbf{s}_{t}\right) \cdot \delta \mathbf{x}_{t}^{m}+\varepsilon_{t}^{m}
$$

where $\delta$ is the fraction of the total ion current that is not modulated, $\mathbf{s}_{t}$ is the $t$-th row of $\mathbf{S}$, and $\mathbf{j}$ is a row vector of length $N$ with all elements equal to 1 . The expectation value of $y_{t}^{m}$ is given by: 


$$
\begin{aligned}
\left\langle y_{t}^{m}\right\rangle & =\sum_{k=1}^{N}(\mathbf{S})_{t k}\left\langle x_{t, k}^{m}\right\rangle+\sum_{k=1}^{N}\left(1-(\mathbf{S})_{t k}\right)\left\langle\delta x_{t, k}^{m}\right\rangle+\left\langle\varepsilon_{t}^{m}\right\rangle \\
& =(1-\delta) \sum_{k=1}^{N}(\mathbf{S})_{t k} p_{k}+\delta \sum_{k=1}^{N} p_{k}+p_{d}
\end{aligned}
$$

Here $(\mathbf{S})_{t k}$ refers to the row $t$, column $k$ element of $\mathbf{S}$, which is also equal to the $k$-th element of $\mathbf{s}_{t}$. The probabilities $p_{k}$ and $p_{d}$ are defined in the Theory section. The MCS outputs the total data vector $\mathbf{y}^{\mathrm{HT}}$, which is the sum of all single-pass data for $M$ passes. This vector is deconvoluted by software with the decoding matrix, $\mathbf{R}$, to obtain the mass spectrum, $\mathbf{z}^{\mathrm{HT}}$.

$$
\mathbf{z}^{\mathrm{HT}}=\mathbf{R} \mathbf{y}^{\mathrm{HT}}=\left(\frac{N+1}{2} \mathbf{S}^{-1}\right) \sum_{m=1}^{M} \mathbf{y}^{m}
$$

The expectation value of the intensity for an ion species $i$ is given by:

$$
\begin{aligned}
\left\langle z_{i}^{\mathrm{HT}}\right\rangle= & \frac{N+1}{2} \sum_{m=1}^{M} \sum_{t=1}^{N}\left(\mathbf{S}^{-1}\right)_{i t}\left\langle y_{t}^{m}\right\rangle \\
= & \frac{N+1}{2} \sum_{m=1}^{M} \sum_{t=1}^{N}\left(\mathbf{S}^{-1}\right)_{i t} \\
& \times\left[(1-\delta) \sum_{k=1}^{N}(\mathbf{S})_{t k} p_{k}+\delta \sum_{k=1}^{N} p_{k}+p_{d}\right] \\
= & \frac{N+1}{2} \sum_{m=1}^{M}\left[(1-\delta) \sum_{k=1}^{N}\left(\sum_{t=1}^{N}\left(\mathbf{S}^{-1}\right)_{t k}(\mathbf{S})_{t k}\right) p_{k}\right. \\
& \left.+\left(\sum_{t=1}^{N}\left(\mathbf{S}^{-1}\right)_{i t}\right)\left(\delta \sum_{k=1}^{N} p_{k}+p_{d}\right)\right] \\
= & \frac{N+1}{2} \sum_{m=1}^{M}\left[(1-\delta) \sum_{k=1}^{N} \delta_{i k} p_{k}+\left(\frac{2}{N+1}\right)\right. \\
& \left.\times\left(\delta \sum_{k=1}^{N} p_{k}+p_{d}\right)\right] \\
= & (1-\delta) \frac{N+1}{2} M p_{i}+\delta \sum_{k=1}^{N} M p_{k}+M p_{d}
\end{aligned}
$$

(b) Conventional TOF (eq 17). For conventional TOF where a periodic pulsing scheme is used, I replaces $\mathbf{S}$ as the encoding matrix and the recorded data at time index $t$ for pass $m$ is given by:

$$
y_{t}^{m}=\mathbf{i}_{t} \cdot \mathbf{x}_{t}^{m}+\left(\mathbf{j}-\mathbf{i}_{t}\right) \cdot \delta \mathbf{x}_{t}^{m}+\varepsilon_{t}^{m}
$$

No deconvolution step is required because the ion counts for an ion species $i, z_{i}^{\mathrm{TOF}}$, are equal to $y_{t}^{\mathrm{TOF}}$, where the ion species $i$ has a flight time corresponding to $t \Delta t$. Thus, the expectation value of $z_{i}^{\mathrm{TOF}}$ is:

$$
\begin{aligned}
\left\langle z_{i}^{\mathrm{TOF}}\right\rangle & =\sum_{m=1}^{M}\left\langle y_{i}^{m}\right\rangle \\
& =\sum_{m=1}^{M}\left[(1-\delta) \sum_{k=1}^{N}(\mathbf{I})_{i k} p_{k}+\delta \sum_{k=1}^{N} p_{k}+p_{d}\right] \\
& =(1-\delta) M p_{i}+\delta \sum_{k=1}^{N} M p_{k}+M p_{d}
\end{aligned}
$$

\section{Equations for the Variance}

(a) HT-TOF (eq 23). The variance of the deconvoluted spectrum $\mathbf{z}$ depends on the variance of the recorded data vector $\mathbf{y}$. The difference between $y_{t}^{m}$ and its expectation value is given by:

$$
\begin{aligned}
y_{t}^{m}-\left\langle y_{t}^{m}\right\rangle= & \sum_{k=1}^{N}(\mathbf{S})_{t k}\left(x_{t, k}^{m}-\left\langle x_{t, k}^{m}\right\rangle\right) \\
& +\sum_{k=1}^{N}\left(1-(\mathbf{S})_{t k}\right)\left(\delta x_{t, k}^{m}-\left\langle\delta x_{t, k}^{m}\right\rangle\right) \\
& +\left(\varepsilon_{t}^{m}-\left\langle\varepsilon_{t}^{m}\right\rangle\right)
\end{aligned}
$$

And, after an $M$-pass acquisition,

$$
\begin{aligned}
y_{t}^{\mathrm{HT}}-\left\langle y_{t}^{\mathrm{HT}}\right\rangle= & \sum_{m=1}^{M}\left[\sum_{k=1}^{N}(\mathbf{S})_{t k}\left(x_{t, k}^{m}-\left\langle x_{t, k}^{m}\right\rangle\right)+\sum_{k=1}^{N}\left(1-(\mathbf{S})_{t k}\right)\right. \\
& \left.\times\left(\delta x_{t, k}^{m}-\left\langle\delta x_{t, k}^{m}\right\rangle\right)+\left(\varepsilon_{t}^{m}-\left\langle\varepsilon_{t}^{m}\right\rangle\right)\right] \quad(\mathrm{A} 12)
\end{aligned}
$$

The variance of $y_{t}^{\mathrm{HT}}$ is calculated as the expectation value of the square of this difference.

$$
\begin{aligned}
\left\langle\left( y_{t}^{\mathrm{HT}}-\right.\right. & \left.\left.\left\langle y_{t}^{\mathrm{HT}}\right\rangle\right)^{2}\right\rangle \\
= & \sum_{m=1}^{M}\left[\sum_{j=1}^{N} \sum_{k=1}^{N}(\mathbf{S})_{t j}(\mathbf{S})_{t k}\left\langle\left(x_{t, j}^{m}-\left\langle x_{t, j}^{m}\right\rangle\right)\left(x_{t, k}^{m}-\left\langle x_{t, k}^{m}\right\rangle\right)\right\rangle\right. \\
& +\sum_{j=1}^{N} \sum_{k=1}^{N}\left(1-(\mathbf{S})_{t j}\right)\left(1-(\mathbf{S})_{t k}\right) \\
& \times\left\langle\left(\delta x_{t, j}^{m}-\left\langle\delta x_{t, j}^{m}\right\rangle\right)\left(\delta x_{t, k}^{m}-\left\langle\delta x_{t, k}^{m}\right\rangle\right)\right\rangle \\
& +\sum_{k=1}^{N}(\mathbf{S})_{t k}\left\langle\left(\varepsilon_{t}^{m}-\left\langle\varepsilon_{t}^{m}\right\rangle\right)\left(x_{t, k}^{m}-\left\langle x_{t, k}^{m}\right\rangle\right)\right\rangle \\
& +\sum_{k=1}^{N}\left(1-(\mathbf{S})_{t k}\right)\left\langle\left(\varepsilon_{t}^{m}-\left\langle\varepsilon_{t}^{m}\right\rangle\right)\left(\delta x_{t, k}^{m}-\left\langle\delta x_{t, k}^{m}\right\rangle\right)\right\rangle \\
& \left.+\left\langle\left(\varepsilon_{t}^{m}-\left\langle\varepsilon_{t}^{m}\right\rangle\right)^{2}\right\rangle\right]
\end{aligned}
$$

This equation can be simplified by calculating the expectation value noting that $\left\langle x_{t, k}^{m}-\left\langle x_{t, k}^{m}\right\rangle\right\rangle=\left\langle\varepsilon_{i}^{m}-\right.$ $\left.\left\langle\varepsilon_{t}^{m}\right\rangle\right\rangle=0$. If $x_{j}^{m}$ and $x_{k}^{m}$ are independent (i.e., $j \neq k$ ), then

$$
\begin{aligned}
& \left\langle\left(x_{t, j}^{m}-\left\langle x_{t, j}^{m}\right\rangle\right)\left(x_{t, k}^{m}-\left\langle x_{t, k}^{m}\right\rangle\right)\right\rangle \\
& \quad=\left\langle x_{t, j}^{m}-\left\langle x_{t, j}^{m}\right\rangle\right\rangle\left\langle x_{t, k}^{m}-\left\langle x_{t, k}^{m}\right\rangle\right\rangle=0
\end{aligned}
$$


and if $j=k$,

$$
\left\langle\left(x_{t, j}^{m}-\left\langle x_{t, j}^{m}\right\rangle\right)\left(x_{t, k}^{m}-\left\langle x_{t, k}^{m}\right\rangle\right)\right\rangle=\left\langle\left(x_{t, k}^{m}-\left\langle x_{t, k}^{m}\right\rangle\right)^{2}\right\rangle
$$

This treatment also applies to $\varepsilon_{t}^{m}$.

$$
\begin{aligned}
& \left.\left\langle\left(\varepsilon_{t}^{m}-\left\langle\varepsilon_{t}^{m}\right\rangle\right)\left(x_{t, k}^{m}-x_{t, k}^{m}\right\rangle\right)\right\rangle \\
& \quad=\left\langle\varepsilon_{t}^{m}-\left\langle\varepsilon_{t}^{m}\right\rangle\left\langle x_{t, k}^{m}-x_{t, k}^{m}\right\rangle\right\rangle=0
\end{aligned}
$$

Using these relations, the equation for the variance simplifies to:

$$
\begin{aligned}
\operatorname{Var}\left(y_{t}^{\mathrm{HT}}\right)= & \left\langle\left(y_{t}^{\mathrm{HT}}-\left\langle y_{t}^{\mathrm{HT}}\right\rangle\right)^{2}\right\rangle \\
= & \sum_{m=1}^{M}\left[\sum_{k=1}^{N}(\mathbf{S})_{t k}^{2}\left\langle\left(x_{t, k}^{m}-\left\langle x_{t, k}^{m}\right\rangle\right)^{2}\right\rangle\right. \\
& +\sum_{k=1}^{N}\left(1-(\mathbf{S})_{t k}\right)^{2}\left\langle\left(\delta x_{t, k}^{m}-\left\langle\delta x_{t, k}^{m}\right\rangle\right)^{2}\right\rangle \\
& \left.+\left\langle\left(\varepsilon_{t}^{m}-\left\langle\varepsilon_{t}^{m}\right\rangle\right)^{2}\right\rangle\right] \\
= & \sum_{k=1}^{N}(\mathbf{S})_{t k}^{2} M p_{k}+\sum_{k=1}^{N}\left(1-(\mathbf{S})_{t k}\right)^{2} M \delta p_{k}+M \sigma_{d}^{2}
\end{aligned}
$$

Here, $p_{k}$ and $\sigma_{d}^{2}$ were substituted for the variances of $x_{t, k}^{m}$ and $\varepsilon_{t}^{m}$, respectively. Because $(\mathbf{S})_{t k}$ can only be 0 or 1 , this equation reduces further by noting that $(\mathbf{S})_{t k}^{2}=(\mathbf{S})_{t k}$. We obtain

$$
\begin{aligned}
\operatorname{Var}\left(y_{t}^{\mathrm{HT}}\right)= & \sum_{k=1}^{N}(\mathbf{S})_{t k} M p_{k} \\
& +\sum_{k=1}^{N}\left(1-(\mathbf{S})_{t k}\right) M \delta p_{k}+M \sigma_{d}^{2}
\end{aligned}
$$

In the deconvoluted spectrum, the difference between the intensity of the $i$-th species $z_{i}^{\mathrm{HT}}$ and its expectation value is represented by:

$$
z_{i}^{\mathrm{HT}}-\left\langle z_{i}^{\mathrm{HT}}\right\rangle=\frac{N+1}{2} \sum_{t=1}^{N}\left(\mathbf{S}^{-1}\right)_{i t}\left(y_{t}^{\mathrm{HT}}-\left\langle y_{t}^{\mathrm{HT}}\right\rangle\right)
$$

The variance of $z_{i}^{\mathrm{HT}}$ is given by the square of the above equation.

$$
\begin{aligned}
\operatorname{Var}\left(z_{i}^{\mathrm{HT}}\right) & =\left\langle\left(z_{i}^{\mathrm{HT}}-\left\langle z_{i}^{\mathrm{HT}}\right\rangle\right)^{2}\right\rangle \\
& =\left(\frac{N+1}{2}\right)^{2} \sum_{t=1}^{N}\left(\mathbf{S}^{-1}\right)_{i t}^{2}\left\langle\left(y_{t}^{\mathrm{HT}}-\left\langle y_{t}^{\mathrm{HT}}\right\rangle\right)^{2}\right\rangle
\end{aligned}
$$

Because the elements of $\left(\mathbf{S}^{-1}\right)_{i t}$ are $\pm 2 /(\mathrm{N}+1)$,

$$
\operatorname{Var}\left(z_{i}^{\mathrm{HT}}\right)=\sum_{t=1}^{N} \operatorname{Var}\left(y_{t}^{\mathrm{HT}}\right)
$$

Substituting for $\operatorname{Var}\left(y_{t}^{\mathrm{HT}}\right)$, the variance of $z_{i}^{\mathrm{HT}}$ becomes

$$
\begin{aligned}
\operatorname{Var}\left(z_{i}^{\mathrm{HT}}\right)= & \sum_{t=1}^{N}\left[\sum_{k=1}^{N}(\mathbf{S})_{t k} M p_{k}\right. \\
& \left.+\sum_{k=1}^{N}\left(1-(\mathbf{S})_{t k}\right) M \delta p_{k}+M \sigma_{d}^{2}\right] \\
= & (1-\delta) \sum_{k=1}^{N}\left(\sum_{t=1}^{N}(\mathbf{S})_{t k}\right) M p_{k} \\
& +\sum_{k=1}^{N} M \delta p_{k}+N M \sigma_{d}^{2} \\
= & {\left[(1-\delta) \frac{N+1}{2}+\delta N\right] \sum_{k=1}^{N} M p_{k}+N M \sigma_{d}^{2} }
\end{aligned}
$$

(b) Conventional TOFMS (eq 27). In conventional TOFMS, the variance of the ion counts in an acquisition bin is independent of the counts in all other bins

$$
\begin{aligned}
y_{t}^{\mathrm{TOF}}-\left\langle y_{t}^{\mathrm{TOF}}\right\rangle= & \sum_{m=1}^{M}\left[\sum_{k=1}^{N}(\mathbf{I})_{t k}\left(x_{t, k}^{m}-\left\langle x_{t, k}^{m}\right\rangle\right)+\sum_{k=1}^{N}(1\right. \\
& \left.\left.-(\mathbf{I})_{t k}\right) \times\left(\delta x_{t, k}^{m}-\left\langle\delta x_{t, k}^{m}\right\rangle\right)+\left(\varepsilon_{t}^{m}-\left\langle\varepsilon_{t}^{m}\right\rangle\right)\right]
\end{aligned}
$$

The variance is calculated by taking the expectation value of the square of the above equation.

$$
\begin{aligned}
\operatorname{Var}\left(y_{t}^{\mathrm{TOF}}\right)= & \left\langle\left(y_{t}^{\mathrm{TOF}}-\left\langle y_{t}^{\mathrm{TOF}}\right\rangle\right)^{2}\right\rangle \\
= & \sum_{m=1}^{M}\left[\sum_{k=1}^{N}(\mathbf{I})_{t k}^{2}\left\langle\left(x_{t, k}^{m}-\left\langle x_{t, k}^{m}\right\rangle\right)^{2}\right\rangle\right. \\
& +\sum_{k=1}^{N}\left(1-(\mathbf{I})_{t k}\right)^{2}\left\langle\left(\delta x_{t, k}^{m}-\left\langle\delta x_{t, k}^{m}\right\rangle\right)^{2}\right\rangle \\
& \left.+\left\langle\left(\varepsilon_{t}^{m}-\left\langle\varepsilon_{t}^{m}\right\rangle\right)^{2}\right\rangle\right] \\
= & \sum_{k=1}^{N}(\mathbf{I})_{t k} M p_{k}+\sum_{k=1}^{N}\left(1-(\mathbf{I})_{t k}\right) M \delta p_{k}+M \sigma_{d}^{2} \\
= & (1-\delta) \sum_{k=1}^{N}(\mathbf{I})_{t k} M p_{k}+\delta \sum_{k=1}^{N} M p_{k}+M \sigma_{d}^{2}
\end{aligned}
$$

Deconvolution is not necessary as the acquisition bin $t$ correspond to species $i$ with flight time $t \Delta t$. Hence,

$$
\operatorname{Var}\left(z_{i}^{\mathrm{TOF}}\right)=(1-\delta) M p_{i}+\delta \sum_{k=1}^{N} M p_{k}+M \sigma_{d}^{2}
$$

\section{References}

1. Brock, A.; Rodriguez, N.; Zare, R. N. Hadamard Transform Time-of-Flight Mass Spectrometry. Anal. Chem. 1998, 70, 37353741.

2. Zare, R. N.; Fernandez, F. M.; Kimmel, J. R. High-Speed Mass Spectrometry Hadamard Transform Time-of-Flight Mass Spectrometry: More Signal, More of the Time. Angew. Chem. Int. Ed. 2003, 42, 30-35.

3. Trapp, O.; Kimmel, J. R.; Yoon, O. K.; Zuleta, I. A.; Fernandez, F. M.; Zare, R. N. Continuous Two-Channel Time-of-flight 
Mass Spectrometric Detection of Electrosprayed Ions. Angew. Chem. Int. Ed. 2004, 43, 6541-6544.

4. Kimmel, J. R.; Fernandez, F. M.; Zare, R. N. Effects of Modulation Defects on Hadamard Transform Time-of-flight Mass Spectrometry (HT-TOFMS). J. Am. Soc. Mass. Spectrom. 2003, 14, 278-286.

5. Kimmel, J. R.; Engelke, F.; Zare, R. N. Novel Method for the Production of Finely Spaced Bradbury-Nielson Gates. Rev. Sci. Instrum. 2001, 72, 4354-4357.

6. Harwit, M. D.; Sloane, N. J. Hadamard Transform Optics; Academic Press: London, 1979, p. 58.

7. Oliver, C. J. Optical Image Processing by Multiplex Coding. Appl. Opt. 1976, 15, 93-106.

8. Tilotta, D. C.; Fry, R. C.; Fateley, W. G. Selective Multiplex Advantage with an Electro-optic Hadamard Transform Spectrometer for Multielemental Atomic Emission. Talanta 1990, $37,53-60$.

9. Tilotta, D. C. Theoretical Multiplex Gain in a UV/VIS Hadamard Transform Spectrometer Utilizing a Uniformly Imperfect Encoding Mask. Talanta 1990, 37, 61-69.

10. Larson, N. M.; Crosmun, R.; Talmi, Y. Theoretical Comparison of Singly Multiplexed Hadamard Transform Spectrometers and Scanning Spectrometers. Appl. Opt. 1974, 13, 2662-2668.

11. Fernandez, F. M.; Vadillo, J. M.; Engelke, F.; Kimmel, J. R.; Zare, R. N.; Rodriguez, N.; Wetterhall, M.; Markides, K. Effect of Sequence Length, Sequence Frequency, and Data Acquisition Rate on the Performance of a Hadamard Transform
Time-of-Flight Mass Spectrometer. J. Am. Soc. Mass. Spectrom. 2001, 12, 1302-1311.

12. Trapp, O., Pearce, E. W., Kimmel, J. R., Yoon, O. K., Zuleta, I. A., Zare, R. N. A Soft On-Column Metal Coating Procedure for Robust Sheathless Electrospray Emitters Used in Capillary Electrophoresis/Mass Spectrometry. Electrophoresis, 2005, 26, 1358-1365.

13. Kimmel, J. R. Continuous, Multiplexed Time-of-Flight Mass Spectrometry of Electrosprayed Ion. Ph.D. Thesis, Stanford University, 2004, pp. 18-46.

14. Lee, H.; Marshall, A. G. Theoretical Maximal Precision for Mass-to-Charge Ratio, Amplitude, and Width Measurements in Ion-Counting Mass Analyzers. Anal. Chem. 2000, 72, $2256-$ 2260.

15. Wineforder, J. D.; Avni, R.; Chester, T. L.; Fitzgerald, J. J.; Hart, L. P.; Johnson, D. J.; Plankly, F. W. A Comparison of Signalto-Noise Ratios for Single Channel Methods (Sequential and Multiplex) Versus Multichannel Methods in Optical Spectroscopy. Spectrochim. Acta 1976, 31B, 1-19.

16. Yoon, O. K. Power Spectral Density of Ion Current from an Electropray Ionization Source. Stanford University, Stanford, CA, unpublished work.

17. Wei, J.; Shui, W.; Zhou, F.; Lu, Y.; Chen, K.; Xu, G.; Yang, P. Naturally and Externally Pulsed Electrospray. Mass Spectrom. Rev. 2002, 21, 148-162.

18. Guilhaus, M.; Selby, D.; Mlynski, V. Orthogonal Acceleration Time-of-Flight Mass Spectrometry. Mass Spectrom. Rev. 2000, 19, 65-107. 